OPEN ACCESS

Edited by:

David Pérez-González,

University of Salamanca, Spain

Reviewed by:

Achim Klug,

University of Colorado, United States

Lars Kunz,

Ludwig Maximilian University

of Munich, Germany

${ }^{*}$ Correspondence:

Mariano Nicolás Di Guilmi

mndiguilmi@dna.uba.ar

Adrián Rodríguez-Contreras

arodriguezcontreras@ccny.cuny.edu

Specialty section

This article was submitted to Auditory Cognitive Neuroscience,

a section of the journal

Frontiers in Neuroscience

Received: 16 January 2021

Accepted: 25 March 2021

Published: 15 April 2021

Citation:

Di Guilmi MN and

Rodríguez-Contreras A (2021)

Characterization of Developmental Changes in Spontaneous Electrical

Activity of Medial Superior Olivary Neurons Before Hearing Onset With

a Combination of Injectable

and Volatile Anesthesia.

Front. Neurosci. 15:654479.

doi: 10.3389/fnins.2021.654479

\section{Characterization of Developmental Changes in Spontaneous Electrical Activity of Medial Superior Olivary Neurons Before Hearing Onset With a Combination of Injectable and Volatile Anesthesia}

\author{
Mariano Nicolás Di Guilmi ${ }^{*}$ and Adrián Rodríguez-Contreras ${ }^{2 *}$ \\ 1 Instituto de Investigaciones en Ingeniería Genética y Biología Molecular, Dr. Héctor N. Torres, INGEBI-CONICET, Buenos \\ Aires, Argentina, ${ }^{2}$ Department of Biology, Center for Discovery and Innovation, City College, Institute for Ultrafast \\ Spectroscopy and Lasers, City University of New York, New York, NY, United States
}

In this work the impact of two widely used anesthetics on the electrical activity of auditory brainstem neurons was studied during postnatal development. Spontaneous electrical activity in neonate rats of either sex was analyzed through a ventral craniotomy in mechanically ventilated pups to carry out patch clamp and multi-electrode electrophysiology recordings in the medial region of the superior olivary complex (SOC) between birth (postnatal day $0, \mathrm{P0}$ ) and P12. Recordings were obtained in pups anesthetized with the injectable mix of ketamine/xylazine ( $K / X$ mix), with the volatile anesthetic isoflurane (ISO), or in pups anesthetized with $\mathrm{K} / \mathrm{X}$ mix that were also exposed to ISO. The results of patch clamp recordings demonstrate for the first time that olivary and periolivary neurons in the medial region of the SOC fire bursts of action potentials. The results of multielectrode recordings suggest that the firing pattern of single units recorded in K/X mix is similar to that recorded in ISO anesthetized rat pups. Taken together, the results of this study provide a framework to use injectable and volatile anesthetics for future studies to obtain functional information on the activity of medial superior olivary neurons in vivo.

Keywords: ketamine, isoflurane, MNTB, in vivo electrophysiology, multi-unit activity

\section{INTRODUCTION}

The accurate organization of neuronal circuits is established during development through activitydependent and activity-independent processes that involve the reorganization and fine-tuning of immature synaptic and cellular networks (Goodman and Shatz, 1993; Hanson and Landmesser, 2004; Kirkby et al., 2013). Before the onset of sensation, spontaneously active cells and signaling mechanisms in sensory organs have been identified as drivers of bursts of neuronal activity that are implicated in activity-dependent refinement of auditory and visual systems in different 
vertebrate species (Maffei and Galli-Resta, 1990; Kotak and Sanes, 1995; Lippe, 1995; Jones et al., 2007; Tritsch et al., 2007; Sonntag et al., 2009; Seabrook et al., 2017; Gribizis et al., 2019). In the auditory system of altricial rodents, calcium action potentials (APs) in cochlear inner hair cells (IHCs) initiate minibursts of APs in auditory neurons before the onset of hearing (Tritsch et al., 2007, 2010; Wang et al., 2015). During the prehearing period, IHCs are transiently innervated by direct axo-somatic efferent synaptic contacts from medial olivocochlear (MOC) neurons located in the brainstem superior olivary complex (SOC; Warr and Guinan, 1979; Simmons et al., 1996). MOC innervation is cholinergic (Glowatzki and Fuchs, 2000; Katz et al., 2004; Gómez-Casati et al., 2005) and mediated by postsynaptic acetylcholine nicotinic receptors (nAChR) containing $\alpha 9$ and $\alpha 10$ subunits (Elgoyhen et al., 1994, 2001; Weisstaub et al., 2002; Lipovsek et al., 2012). The nAChR is coupled to the activation of small-conductance calcium-activated SK2 potassium channels expressed in the IHCs, which mediate the hyperpolarization of IHC membrane potential in response to MOC efferent activation (Glowatzki and Fuchs, 2000). Thus, it has been proposed that MOC efferent-mediated inhibition might contribute to pattern trains of IHC calcium APs during the critical developmental period preceding hearing onset (Kros et al., 1998; Glowatzki and Fuchs, 2000; Marcotti et al., 2003; Johnson et al., 2011; Sendin et al., 2014; Moglie et al., 2018).

In recent years, different lines of genetically manipulated mouse models were used to study how modulation of the cochlear pacemaker affects the maturation of central auditory neurons and synapses. In the brainstem medial nucleus of the trapezoid body (MNTB), electrophysiological techniques were employed in slices and in vivo recordings of anesthetized $\alpha 9 \mathrm{nAChR}$ mutant mouse pups (Clause et al., 2014; Di Guilmi et al., 2019). Despite this progress, we identified a major discrepancy in published studies based on the use of different types of anesthetics in different rodent species. The two most widely used anesthetics in brainstem studies are the injectable mix of ketamine/xylazine (K/X mix) in mice (Sonntag et al., 2009; Clause et al., 2014; Di Guilmi et al., 2019), and the volatile anesthetic isoflurane (ISO) in rats (Tritsch et al., 2010; Crins et al., 2011; Sierksma and Borst, 2017). Although different factors are taken into account to choose the type of anesthetic, few studies have addressed its potential effects on in vivo spontaneous neuronal activity. For example, although previous studies have identified burst firing units in mice and rats (Sonntag et al., 2009; Tritsch et al., 2010; Clause et al., 2014), only one study in ISO-anesthetized rats reported regular firing units (Tritsch et al., 2010). This raises the possibility that the firing pattern of MNTB and other auditory brainstem neurons could be affected by the type of anesthetic used. Recent works in awake animals do not settle this issue, because optical calcium reporter fluorescence techniques were employed in auditory midbrain and cortex (Babola et al., 2018, 2020; Wang H.-Y. et al., 2020), and when electrophysiology experiments were used, they were performed in the somatosensory cortex of awake mice (Wang Y. et al., 2020).

In this work, we studied neuronal activity with electrophysiology techniques for single unit recording and ensemble multi-unit recording in the SOC of neonate rats.
We used K/X mix to induce and maintain rat pups in a stable anesthetized state, while the percent ISO was titrated with precision to study its inhibitory effects on single unit and multiunit activity of medial superior olivary neurons. We also used the two anesthetics independently to monitor the overall activity of a population of neurons in animals at different postnatal ages, and ISO to identify the location of neurons with different firing properties. We found that burst and regular firing units are present in $\mathrm{K} / \mathrm{X}$ mix anesthetized rat pups, that when ISO is added to $\mathrm{K} / \mathrm{X}$ mix anesthetized pups it has inhibitory effects on the two types of units, and that some regular firing cells seem to be resistant to the inhibitory effects of maximal doses of ISO. Our results also demonstrate that despite the suppressive effects of anesthetics, the ensemble electrical activity of superior olivary neurons recorded in $\mathrm{K} / \mathrm{X}$ mix or in ISO-anesthetized rat pups ramps up during development.

\section{MATERIALS AND METHODS}

\section{Animal Housing and Breeding}

The Institutional Animal Care and Use Committee of the City College of New York specifically reviewed and approved this study. The cohorts of adult Wistar rats used in this study were obtained from a commercial supplier at postnatal age 65 (P65, Charles River). Breeding trios of one male and two females were set for 5 days. At the completion of the breeding period, males were removed from the study while females were housed in pairs for 14 more days, and then individually until they gave birth. Pups were used from the day of birth (P0) to 12 days after birth (P12). Experiments were designed to minimize the number of animals used.

\section{Ventral Surgery and Brain Processing}

A total of 85 pups from 37 litters were prepared for surgery to expose the ventral skull for electrophysiology experiments. The surgical procedures used are those described by RodríguezContreras et al. (2008), with minor modifications. Neonate rat pups were initially anesthetized by an intraperitoneal injection of a mixture of ketamine hydrochloride $(0.1 \mathrm{mg} / \mathrm{g}$ body weight; Ketathesia) and xylazine hydro-chloride $(0.005 \mathrm{mg} / \mathrm{g}$ body weight; Anased), here referred as $\mathrm{K} / \mathrm{X}$ mix. After anesthesia induction, animals were tracheotomized, intubated, and mechanically ventilated using a MiniVent type 845 ventilator (Harvard Apparatus). During surgery, anesthesia was carefully monitored on the basis of pedal reflexes, and maintained by supplementary injections of one-third of the initial dose of $\mathrm{K} / \mathrm{X}$ mix as necessary (Sonntag et al., 2009). For the subset of experiments described in Figure 2, animals were initially anesthetized inside an induction chamber with $3 \%$ isoflurane (ISO) carried in oxygen, and maintained anesthetized with $1.5 \%$ ISO carried in oxygen, delivered via a nose cone or a direct connection to the mechanical ventilator. A small ventral craniotomy $(1.5 \times 1.5 \mathrm{~mm})$ was performed on each pup, and the brain vascular landscape constituted by the basilar artery and the anterior inferior cerebellar artery was exposed. The dura was carefully removed prior to recording. Body temperature 
was controlled during surgery and recording with a heating pad set to $37^{\circ}$ (FHC, Maine, United States). After successful recordings were obtained, animals received an overdose injection of Euthasol, and were removed from the setup to be perfused with a solution of $4 \%$ paraformaldehyde in $0.1 \mathrm{M}$ phosphate buffer. Brains were removed from the skull and processed for histology to be sectioned in $60 \mu \mathrm{m}$ thick slices. Brainstem slices were mounted onto glass slides and counterstained with DAPI prior to applying mounting medium and a glass coverslip. Brain sections were imaged with an LSM800 confocal microscope located at the CCNY imaging core facility.

\section{Patch Clamp Recordings}

Recordings were made at a depth of 200-600 $\mu \mathrm{m}$ from the pial surface using borosilicate glass pipettes (5-8 M 2 ) filled with artificial cerebrospinal fluid (ACSF) containing (in $\mathrm{mM}$ ): $125 \mathrm{NaCl}, 5 \mathrm{KCl}, 2 \mathrm{MgSO}_{4}, 2 \mathrm{CaCl}_{2}, 10 \mathrm{D}$-glucose, 10 HEPES, adjusted to $\mathrm{pH} 7.4$ with $\mathrm{NaOH}$, or intracellular solution containing (in $\mathrm{mM}$ ): $126 \mathrm{~K}$-gluconate, $20 \mathrm{KCl}, 10$ $\mathrm{Na}_{2}$ - phosphocreatine, $4 \mathrm{MgATP}, 0.3 \mathrm{Na}_{2} \mathrm{GTP}, 0.5$ EGTA, and 10 HEPES $\left(310 \mathrm{mmol} . \mathrm{kg}^{-1}\right)$, adjusted to $\mathrm{pH} 7.2$ with $\mathrm{KOH}$. Extracellular potentials were detected in the loose-patch configuration, while intracellular potentials were recorded in the whole-cell configuration. Pipettes were advanced from the brain surface in large steps of $50 \mu \mathrm{m}$ to a depth of $200 \mu \mathrm{m}$ using high positive pressure (300 mbar). When in cell search mode, pressure was reduced to $30 \mathrm{mbar}$ and the pipette was moved in 1.5 or $2 \mu \mathrm{m}$ steps. The pipette tip resistance was monitored with a $10 \mathrm{mV}$ test pulse in voltage clamp or when a shift in the DC was noted in current clamp. When the tip resistance increased suddenly, suggesting that a putative cell was encountered, pressure was released and slight negative pressure of 30-50 mbar was applied until a low resistance seal was formed (10-50 M $\Omega$ ) for loosepatch recordings, or until a high resistance seal was formed (1-10 G $\Omega$ ) for whole-cell recordings. High-resistance seals were broken by applying fast bouts of negative pressure. Whole-cell recordings were not corrected for the liquid junction potential. Voltage was recorded with pClamp9 software using an Axopatch 200B amplifier, filtered at $2-5 \mathrm{kHz}$ and sampled at $20 \mathrm{kHz}$ with a Digidata 1440B (MDS Analytical Technologies). Inclusion of Alexa Fluor 594 (1\%, Invitrogen) in the pipette solution allowed post hoc confirmation of recording sites or recorded cells in paraformaldehyde-fixed brainstem sections.

\section{Multi-Electrode Recordings}

Prior to recording silicon probes (polytrode $4 \times 4$ 16-channel arrays, NeuroNexus, A4 × 4-3 mm-50-125-177-A16; 1 M $\Omega$ ) were coated with the lipophilic dye DiI (Invitrogen) to confirm proper targeting to the MNTB and medial region of the SOC in post hoc histological analysis. Recordings were made by placing probes with the medial-most shank at coordinates of 300-600 $\mu \mathrm{m}$ rostral and $300 \mu \mathrm{m}$ lateral to the branch point of the anterior cerebellar artery from the basilar artery, and at a depth of 400$500 \mu \mathrm{m}$ from the pial surface. All data from silicon probe recordings was acquired in blocks of $10 \mathrm{~min}$, sampled at $24.4 \mathrm{kHz}$, amplified and digitized in a single-head stage (TDT system III hardware, Tucker-Davis Technologies, Alachua, FL).

\section{Effect of Isoflurane on $\mathrm{K} / \mathrm{X}$ Mix Anesthetized Pups}

ISO was delivered in medical grade oxygen gas via a custom-made tube that connected a vaporizer to the animal ventilator at a rate of $1 \mathrm{~L} \mathrm{~min}^{-1}$. For single-unit recordings three protocols of ISO delivery were used. The first protocol consisted of acquiring 5 blocks of $10 \mathrm{~min}$ each to obtain a baseline, two consecutive blocks in $1.5 \%$ isoflurane, and two washout blocks. The second was a staircase protocol that consisted of a single $10 \mathrm{~min}$ long recording where APs were quantified in six $100 \mathrm{~s}$ bins: baseline (0\% ISO), $1.5 \%$ ISO, 3.0\% ISO, $4.5 \%$ ISO and two washout bins (0\% ISO). A third protocol was used for units that showed resistance to the staircase protocol and consisted of six $100 \mathrm{~s}$ bins: baseline, three bins at 5\% ISO, and two washout bins. The effect of ISO was evaluated in multi-electrode recordings by using a procedure similar to the first protocol described above, except that between the baseline recording and the first two blocks in 1.5\% ISO, a $90 \mathrm{~s}$ period in $1.5 \%$ ISO was added.

\section{Data and Statistical Analyses}

Unless indicated, data is presented as mean \pm sem. In all cases, $n$ indicates the number of units or cells tested, with the exception of Figure 3 where $n$ indicates the number of animals. Extracellular single-unit data was filtered in two steps. First, to eliminate 60 cycle electrical interference $(0.6 \mathrm{~Hz}, 3 \mathrm{~dB}$ bandwidth); and second, with a high-pass Bessel (8-pole) filter with a $-3 \mathrm{~dB}$ cutoff of $5 \mathrm{~Hz}$ using Clampfit 10.6 (Molecular Devices). All data was analyzed using NeuroMatic in Igor Pro software (WaveMetrics; Rothman and Silver, 2018). APs were detected using a positive threshold set to six times the standard deviation of the baseline noise. Single-unit recordings where an obvious gradual decrease or rundown in spike amplitude occurred were excluded from analysis. Multielectrode multi-unit data was exported as NEX files for analysis in Plexon software (Offline sorter 4.4 and Neuroexplorer). APs were detected using a negative threshold set to six times the standard deviation of the baseline noise. Multiunit activity (MUA) collected in every channel was considered. All statistical tests were performed with Statistica 7.0 software (Stat Soft; RRID:SCR_014213). Non-parametric Kruskall-Wallis (KW) test was used. Values of $p<0.05$ were considered significant.

\section{Drugs and Reagents}

All drugs and reagents were purchased from Sigma-Aldrich (RRID:SCR_008988).

\section{RESULTS}

\section{Isoflurane Inhibits Burst and Regular Firing Units in the Ventral Brainstem of K/X Mix-Anesthetized Rat Pups}

Single-unit recordings were performed in five $\mathrm{K} / \mathrm{X}$ mixanesthetized rat pups before hearing onset, and the effects of $1.5 \%$ isoflurane were evaluated on the firing properties of six single-units (one unit from a P3 pup and five units from four P6 pups). Two of the units showed burst firing 
and four units showed regular firing patterns under baseline conditions (exemplar recordings are shown in Figures 1A-C). These recorded units had variable responses to ISO. The activity of the two burst firing units was inhibited to 96 and $99.6 \%$ compared to baseline and did not recover during washout. The activity of three out of the four regular firing units was partially inhibited to $34 \pm 21 \%$ compared to baseline, and only in one unit recovered to baseline levels during washout. Lastly, one of the four regular firing units slightly increased its activity by $2 \%$ in ISO compared to baseline, but its activity was suppressed to $19 \%$ during washout. These inhibitory and potentiating effects of ISO on single-unit firing properties were observed in the interspike-interval histograms shown in Figures 1D-F. Small changes in the waveform of recorded units were also noted (insets in Figures 1D-F).

To address the variation of $1.5 \%$ ISO effects on recorded units, additional experiments were performed on thirteen single-units recorded from two P3 pups, two P6 pups, and three P9 pups by increasing the ISO dose from 0 to $4.5 \%$ in $1.5 \%$ steps. It was found that two P3 units, two P6 units and four P9 units showed clear signs of dose-dependent inhibition at $4.5 \%$ ISO compared to baseline. These cells did not recover during the washout period (Figure 1G). In a total of five additional single-units from pups of all ages, evidence of potentiation or resistance to $4.5 \%$ ISO was found. In three of these units, a small inhibitory effect was observed during the washout period (Figure 1H). Three of these
A

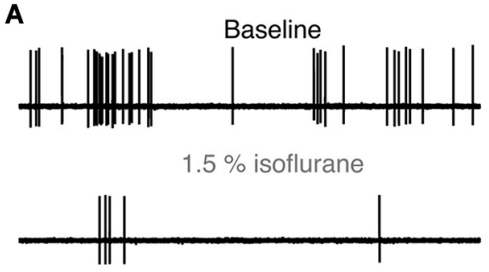

Washout
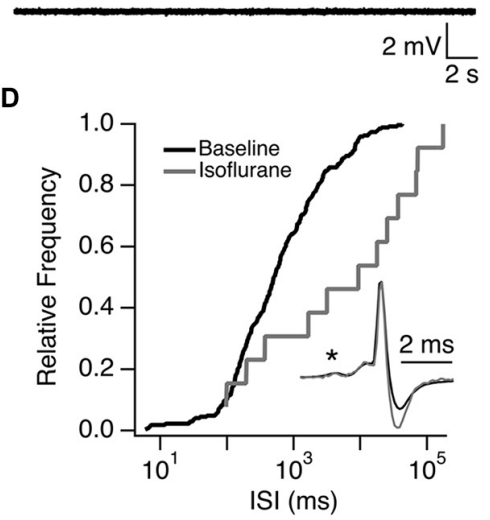

G

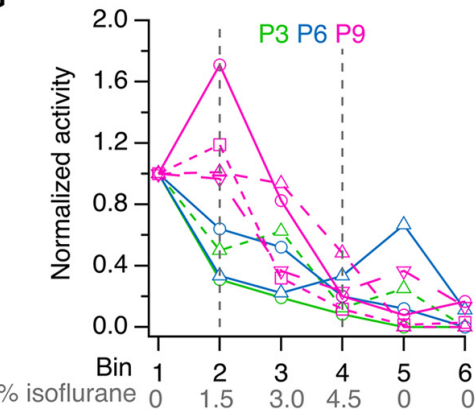

B
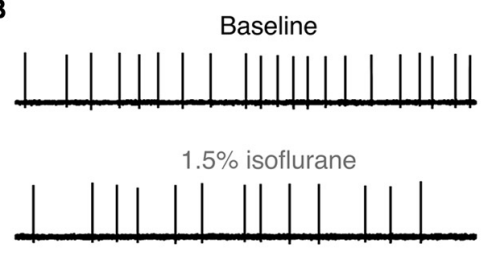

Washout

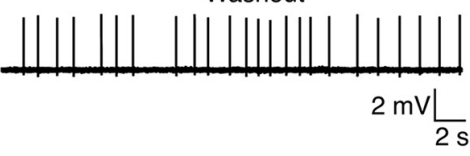

E

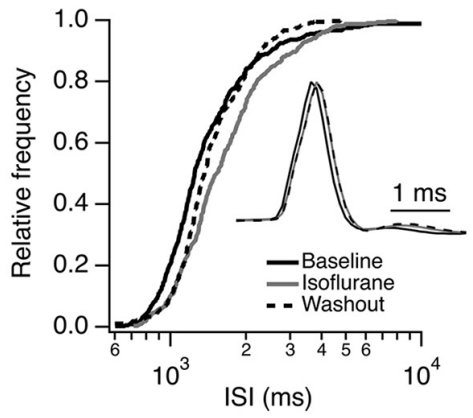

H

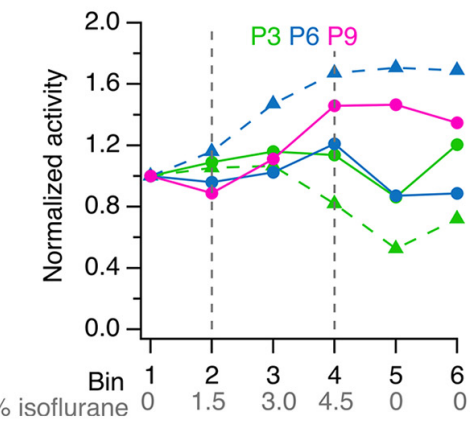

C

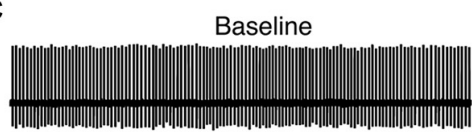

$1.5 \%$ isoflurane

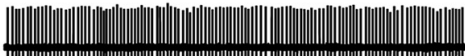

Washout
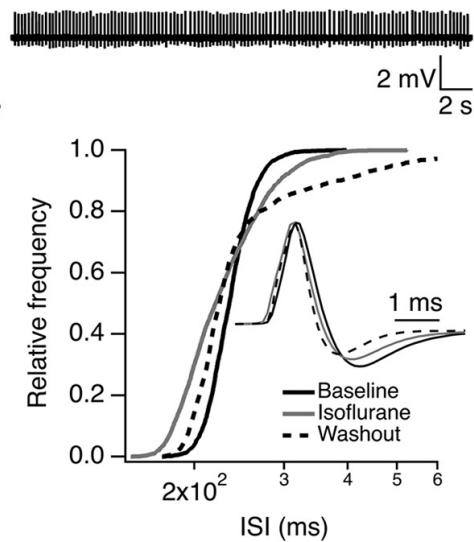

I

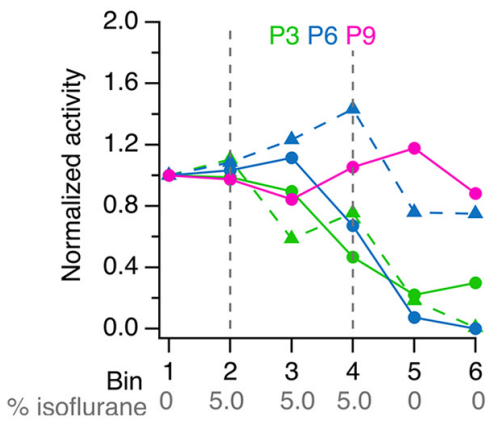

FIGURE 1 | ISO inhibit single unit activity in KX mix anesthetized pups. (A) Burst firing unit from a P6 rat. (B) Regular firing unit from a P3 rat. (C) Regular firing unit from a P6 rat. (D) Effect of ISO on the inter-spike interval (ISI) distribution of single unit shown in (A). (E) Effect of ISO on ISI distribution of single unit shown in (B). (F) Effect of isoflurane on ISI distribution of single unit shown in (C). (G) Inhibitory effect of ISO on single unit activity recorded at P3 (green), P6 (blue) and P9 (magenta). (H) ISO resistant single units recorded at P3, P6 and P9. (I) Exposure to $5 \%$ ISO inhibited 3 of 5 single units shown in (H). ISI = inter-spike interval. Asterisk in D indicates the pre-spike in this complex waveform. Inset waveforms in (D) are averages of 269 and 11 action potentials in baseline and isoflurane, respectively. Inset waveforms in (E) are averages of 363, 330, and 392 action potentials in baseline, isoflurane and washout, respectively. Inset waveforms in (F) are averages of 2,538, 2,599, and 2,046 action potentials in baseline, isoflurane and washout, respectively. Lines and symbols in (H,I) identify the same single units under different isoflurane conditions. Vertical dashed lines in (G-I) indicate the onset and offset of isoflurane application. 
units were inhibited by prolonged exposure to $5 \%$ ISO and did not recover during the washout period. The remaining two units from P6 and P9 pups showed signs of resistance and potentiation even at such high dosage (Figure 1I).

Altogether, these experiments show that burst and regular firing cells are present in $\mathrm{K} / \mathrm{X}$ mix-anesthetized rat pups, that most burst and regular firing units are inhibited permanently by ISO, while some regular firing units appear to be resistant to inhibition and show indications of potentiation after ISO is delivered to $\mathrm{K} / \mathrm{X}$ mix-anesthetized rat pups. Resistance and potentiation to ISO was observed more frequently in P6 and $\mathrm{P} 9$ rat pups.

\section{Heterogeneous Population of Single-Units: Inactive Versus Active Neurons}

It was noted that from a total of nineteen recorded single-units, five units fired in a burst type pattern, while the rest fired in a regular spike pattern. Furthermore, three of the burst firing units did not show complex waveforms adjudicated to MNTB neurons. These observations raised the possibility that other SOC neurons may fire burst type or regular type patterns. One caveat of looseseal patch clamp recordings is that cells cannot be identified, unless iontophoretic methods are used to label them (Pinault, 1996; Cid and de la Prida, 2019). As an alternative to identify the morphology and location of recorded cells, whole-cell current clamp recordings were performed in forty ISO anesthetized pups between ages P0 and P11 (Figure 2). Active and inactive cells were identified in current clamp recordings. Active cells fired APs in burst $(n=10)$ or regular type $(n=5)$ patterns, while inactive cells did not fire APs at rest ( $n=25$; Figure 2A). Two kinds of inactive cells were further identified based on their response to depolarizing current injection. Some cells fired APs $(n=13)$, while other cells did not $(n=12$; Figure $2 \mathbf{B})$. Therefore, inactive cells were classified as able or unable to fire APs (Figure 2C). After histological processing, the morphology and location of twenty-three recorded cells was confirmed in the medial region of the SOC (Figure 2D). Eight burst firing cells were observed within and outside the boundaries of SOC nuclei such as the MNTB and the MSO/SPN; two regular spiking cells were observed in the dorsal region outside the boundary of the MNTB; two inactive cells were observed within the boundaries of the MNTB; and eleven inactive cells were found at ventral, medial, lateral and dorsal locations outside the boundaries of the MNTB and MSO/SPN.

In sum, these experiments show that ISO-anesthetized rat pups have active and inactive neurons in the SOC. Several cells located in the medial region of the SOC fire APs in burst or regular patterns. However, while burst-firing cells were observed within and outside the boundaries of SOC nuclei, regular firing cells were observed only outside the boundaries of SOC nuclei. Some inactive cells are capable of firing action potentials upon depolarization of their membrane potential, while others cannot fire action potentials suggesting irreversible inhibition or a different cellular phenotype (i.e., non-neuronal glial).

\section{Multiunit Activity Increases During Postnatal Development}

Next, the effect of the injectable and volatile anesthetics on the ensemble activity of the medial SOC was evaluated separately
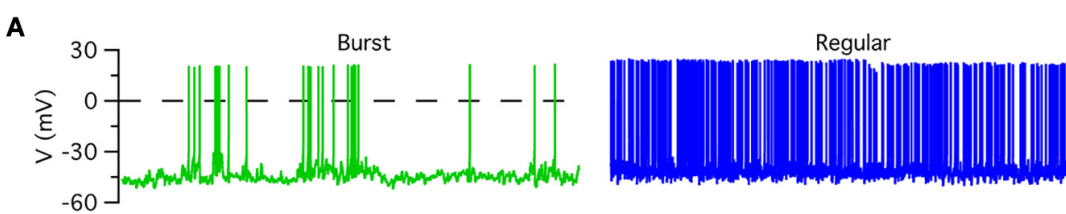

B

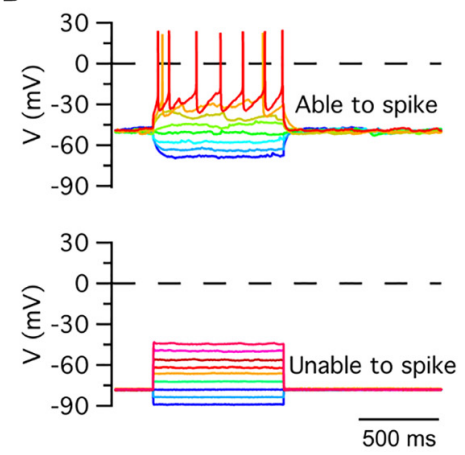

C

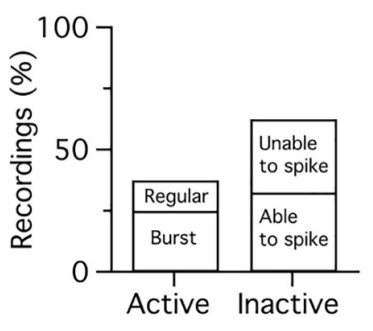

D

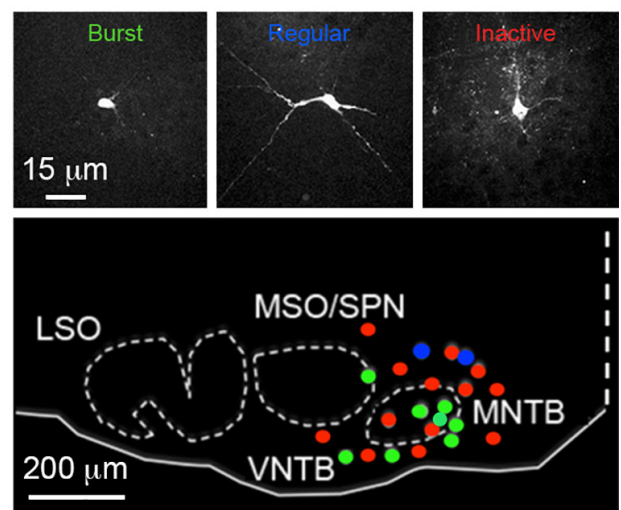

FIGURE 2 | Heterogeneous electrical activity in medial olivocochlear neurons. (A) Exemplar patch-clamp whole cell recordings of burst firing, regular firing and inactive cells obtained from pups anesthetized with 1.5\% ISO. (B) Inactive cells were subjected to hyperpolarizing and depolarizing voltage steps. Depolarization steps triggered action potentials in some cells but not in others. (C) Percent of active and inactive cells. (D) Exemplar images of cells shown in (A) (top row). Distribution of recorded cells in the auditory brainstem in neonate pups (bottom). Recording traces were not corrected for the liquid junction potential. 
with multi-electrode probes in pups aged between P0 and P12 ( $n=29$; Figure 3A). Despite the large variability of spontaneous MUA within each developmental group, the overall MNTB spontaneous activity ramped up during development under $\mathrm{K} / \mathrm{X}$ mix $(n=14$; Figures $3 \mathrm{~B}, \mathrm{C}$, left panel) or ISO ( $n=15$; Figures $3 B, C$ right panel). It is important to note that the mean firing rate was similar under both conditions. Statistical analysis showed only differences under ISO displaying lower activity at P0 compared to P9 (K-W, $p=0.04$; multiple comparisons, $p<0.05$; $n=3$ ). Taken together, these results suggest that the overall level of spontaneous electrical activity increases during postnatal development and that the maximal ensemble firing rate was similar between the two anesthetics.

\section{Developmental Profile of ISO Inhibition}

To examine whether ISO may act over the anesthetic effect of $\mathrm{K} / \mathrm{X}$ mix and considering that both anesthetics have different synaptic targets (MacDonald et al., 1987; Wang Y. et al., 2020), we implemented the recording protocol illustrated in Figure 4A. Figure 4B displays three examples of MUA at different developmental ages (P3, P6, and P9) along the experimental protocol. In all cases, application of $1.5 \%$ ISO depressed the spiking activity, and a partial or total washout could be observed. The activity level was quantified after application of $1.5 \%$ ISO relative to K/X mix baseline (Figure 4C). P0 was excluded from the statistical analysis due to the activity being too small and the estimation of inhibition may not be reliable. Statistical analysis showed that ISO at $10 \mathrm{~min}$ had a greater initial inhibition at P6 in comparison with $\mathrm{P} 12$ (K-W, $p=0.007$; multiple comparisons, $p<0.05 ; n=3)$. In conjunction with loose-seal patch clamp recordings (Figure 1), these experiments show that the inhibitory effect of ISO is heterogeneous before hearing onset.

\section{DISCUSSION}

General anesthetics are widely used for surgical procedures in animal models and sometimes are required during in vivo experiments. For example, recent studies have targeted dorsally accessible brain structures like the inferior colliculus (IC) and auditory cortex (AC) in awake transgenic mice that had been exposed to anesthetics during preparatory surgery

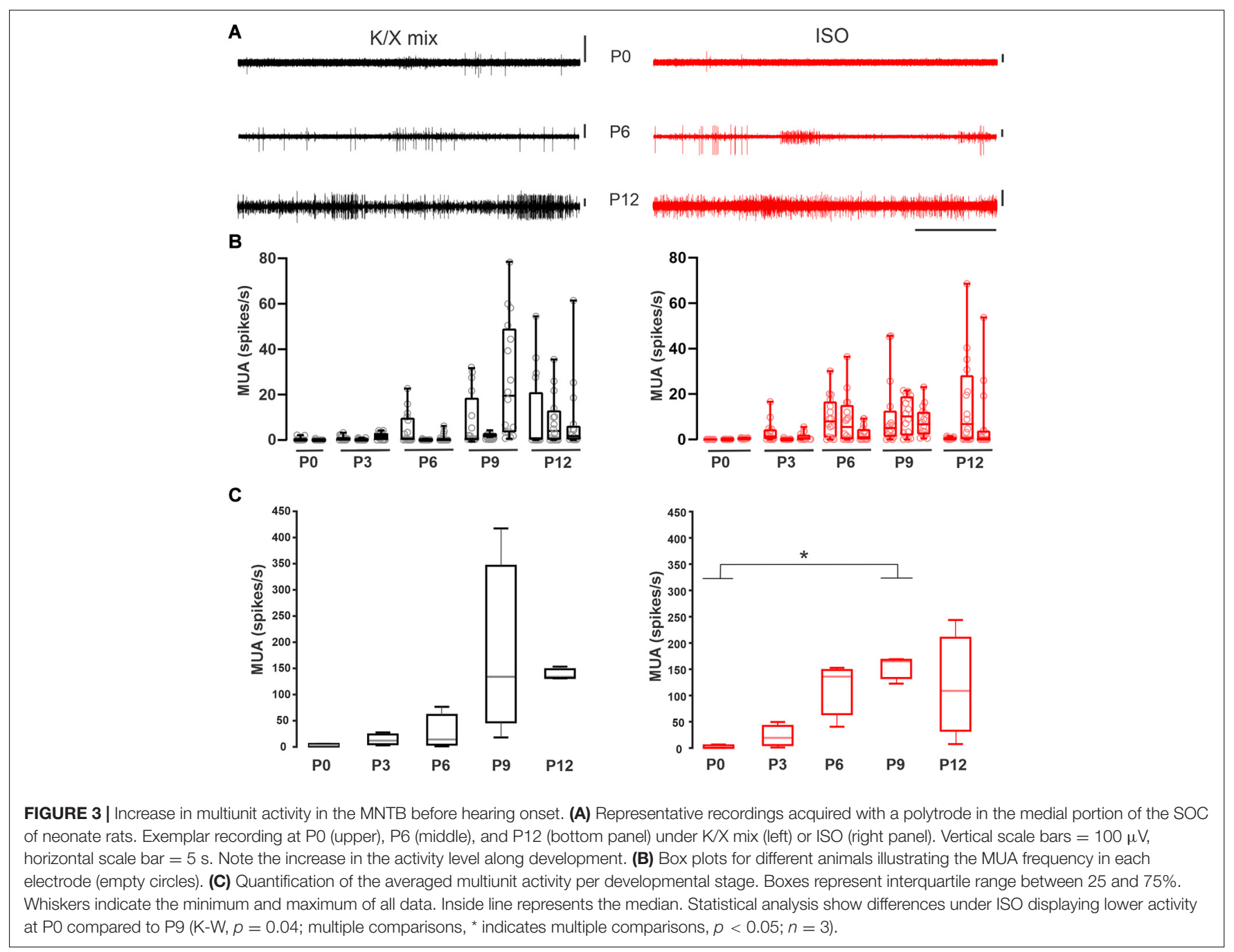




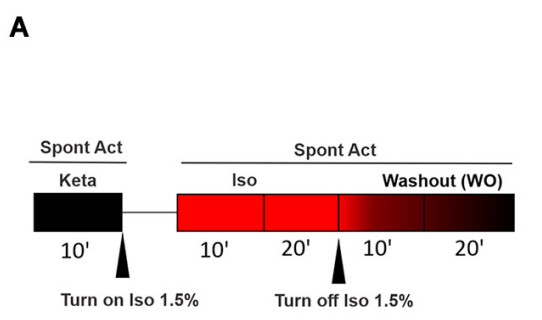

\section{C}

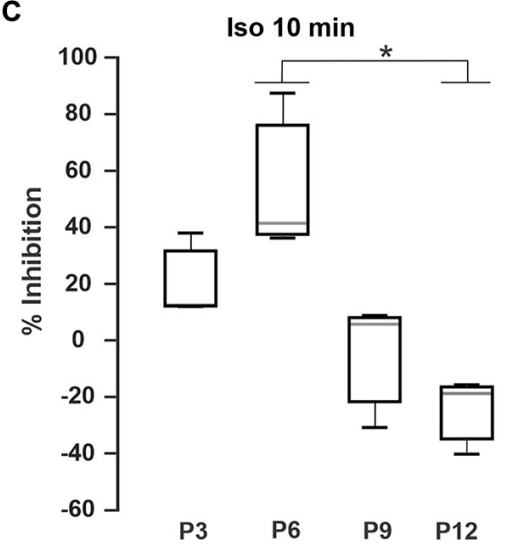

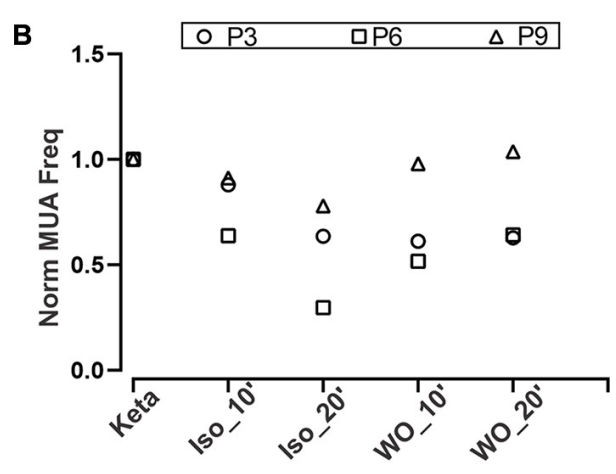

Iso $20 \mathrm{~min}$

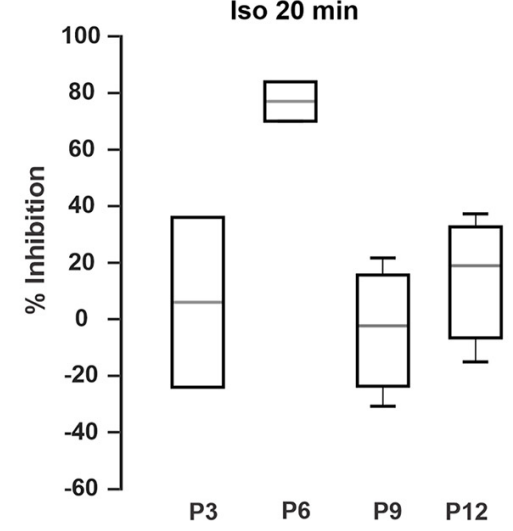

FIGURE 4 | Time-dependent inhibition of Isoflurane. (A) Schematic diagram of the pharmacological protocol for testing Isoflurane inhibition. The first step was to record the spontaneous activity during $10 \mathrm{~min}$ under $\mathrm{K} / \mathrm{X}$ mix (black box). Then we applied Isoflurane $1.5 \%$ for $90 \mathrm{~s}$ followed by two recording rounds of $10 \mathrm{~min}$ to obtain MUA after 10 or 20 min, respectively (red boxes). After that, ISO was turned off to evaluate the washout for the next 20 min. (B) Three examples showing the ISO inhibition and its washout at different developmental ages (P3, P6, and P9). Normalized MUA frequency relative to the K/X mix state (Norm MUA freq).

(C) Percentage of Inhibition (MUA frequency under ISO/MUA frequency under K/X mix) after 10 (left) or 20 min (right) with ISO at different developmental stages.

Statistical analysis show that ISO at 10 min had a greater inhibition at P6 in comparison with P12 (K-W, $p=0.007$; multiple comparisons, ${ }^{*}$ indicates multiple comparisons, $p<0.05 ; n=3)$.

(Babola et al., 2018, 2020). However, if ventrally accessible brainstem structures are targeted to perform imaging and/or electrophysiological recordings, a general anesthetic must be used to perform recordings in animals that are mechanically ventilated (Rodríguez-Contreras et al., 2014; Di Guilmi et al., 2019), or that are immobilized to facilitate accurate electrode targeting (Sonntag et al., 2009; Clause et al., 2014). In this work we studied the impact of K/X mix and ISO on the spontaneous electrical activity of medial superior olivary neurons in neonate rodents.

This study provides three main findings that are relevant to understand the role of medial superior olivary neurons in auditory system development. First, to the best of our knowledge, this is the first study that compares K/X mix and ISO in the same rodent species. The firing pattern of single units recorded in $\mathrm{K} / \mathrm{X}$ mix was similar to that reported in ISO anesthetized rat pups (Figure 1; Tritsch et al., 2010). Furthermore, this is the first study that identified burst-firing neurons outside the MNTB, which due to their location were mapped to the medial portion of the SOC (Figure 2). Second, it was found that ISO displayed a large scatter on its suppressing effects on electrical activity of auditory brainstem neurons. In pups that were pre-anesthetized with $\mathrm{K} / \mathrm{X}$ mix, around two thirds of recorded cells were inhibited by
1.5\% isoflurane, the isoflurane EC50 estimated under controlled conditions (Figures 1, 4; Wang Y. et al., 2020). However, an estimated one third of recorded units, all of which had a regular firing pattern, were resistant to $1.5 \%$ and were inhibited by $5 \%$ isoflurane delivered over $20 \mathrm{~min}$. Third, this work showed that electrical ensemble activity of medial superior olivary neurons ramps up during development before hearing onset, with a similar developmental profile under both anesthetics (Figure 3).

One caveat of the present study is that we were not able to study the effect of different concentrations of $\mathrm{K} / \mathrm{X}$ mix on auditory brainstem neuronal activity. Future studies could first induce and maintain an anesthetic state with ISO, while K/X mix is delivered and titrated with precision via intravascular infusion. Another limitation of the present study is that the spatial location of neurons with different firing properties was inferred after intense post hoc histological analysis, and the functional properties of those cells could not be studied in the same animal. We consider that our results shed light on the use of anesthetics as tools for future studies that enable patch-clamp electrophysiology combined with imaging studies of medial superior olivary neurons in vivo. The approach would be to use $\mathrm{K} / \mathrm{X}$ mix to induce a stable anesthetic state and ISO to probe 
neuron inhibition on burst and regular firing neurons, which are predicted to have different responses to ISO. A combined optical calcium reporter imaging and electrophysiology approach would be desirable and useful to study MOC neurons, whose cell bodies are diffusely located in the ventral nucleus of the trapezoid body (VNTB) (Warr, 1975; Warr and Guinan, 1979; Guinan et al., 1983) and could be labeled with fluorescent reporters using genetic approaches (Cadenas et al., 2020).

Lastly, it is important to consider how the present study may advance our understanding of the mechanisms of anesthetic action throughout life, since neurons in the medial superior olive have also been studied in adult rodents anesthetized with $\mathrm{K} / \mathrm{X}$ mix to investigate auditory processing (Lorteije et al., 2009; van der Heijden et al., 2013). It is noted that the K/X mix and ISO have different synaptic targets [i.e., postsynaptic NMDA receptors (MacDonald et al., 1987) or presynaptic effect (Wang Y. et al., 2020), respectively]. In agreement with previous reports, synaptic depressant percentage depended on ISO concentration and the developmental age of pups (Wu et al., 2004; Wang Y. et al., 2020). Different reasons related to the plastic changes of auditory synapses (Wu et al., 2004), the timing of hearing onset (Qiu et al., 2020), and the fact that the principal target of ketamine is the NMDA receptor (MacDonald et al., 1987) which declined to very low levels at the end of the second postnatal week (Taschenberger and von Gersdorff, 2000; Joshi and Wang, 2002), may explain the results of this study. Inhaled anesthetics diffuse from the alveoli into arterial and capillary blood, and are assumed to equilibrate rapidly with the wellperfused central nervous system (Hemmings et al., 2005). Thus, the heterogeneity of inhibitory effects observed in the results of this study, may also be explained by the hypothesis that the physical and functional properties of the vasculature, the diffusive properties of the extracellular space between vessels and neurons, and the clearance mechanisms of extracellular fluid available during this developmental window may be important factors that determine the access of anesthetics to target neurons in the brain (Nicholson et al., 2000; Shi and Rodríguez-Contreras, 2016; Mestre et al., 2020). A major difference in the effects of ISO in adult animals compared to neonates is the induction of slow waves of neuronal activity, an effect not described in neonates (Golshani et al., 2009; Brier et al., 2019). In this study, we observed that a group of recorded cells were inactive. One of the responsible factors of neuronal excitability is the resting membrane potential $(\mathrm{Vm})$. Volatile anesthetics induced negative potentials on cortical cells (Petersen et al., 2003) and the hyperpolarization of postsynaptic MNTB neurons in brain slices

\section{REFERENCES}

Babola, T. A., Kersbergen, C. J., Wang, H. C., and Bergles, D. E. (2020). Purinergic signaling in cochlear supporting cells reduces hair cell excitability by increasing the extracellular space. Elife 9, 1-30. doi: 10.7554/eLife.52160

Babola, T. A., Li, S., Gribizis, A., Lee, B. J., Issa, J. B., Wang, H. C., et al. (2018). Homeostatic Control of Spontaneous Activity in the Developing Auditory System. Neuron 99, 511-524. doi: 10.1016/j.neuron.2018.07.004

Brier, L. M., Landsness, E. C., Snyder, A. Z., Wright, P. W., Baxter, G. A., Bauer, A. Q., et al. (2019). Separability of calcium slow waves and
(Wang Y. et al., 2020). Rusu and Borst (2010) demonstrated in slices containing the MNTB that Vm decreases during the first postnatal week becoming progressively more negative. During the first two postnatal weeks, the heterogeneity in the intrinsic properties maturation is larger than in adulthood, and probably it is a substrate for our observations. However, the effect of ISO on the Vm of these cells is under debate (Wu et al., 2004; Wang Y. et al., 2020) and a further study of molecular mechanisms during development is necessary to clarify the results.

\section{DATA AVAILABILITY STATEMENT}

The raw data supporting the conclusions of this article will be made available by the authors, without undue reservation.

\section{ETHICS STATEMENT}

The animal study was reviewed and approved by the Institutional Animal Care and Use Committee of the City College of New York.

\section{AUTHOR CONTRIBUTIONS}

AR-C designed and performed the patch-clamp, multielectrode, and histology experiments. MD designed and performed the multi-electrode experiments. $\mathrm{MD}$ and $\mathrm{AR}-\mathrm{C}$ analyzed the data and wrote and edited the manuscript. Both authors contributed to the article and approved the submitted version.

\section{FUNDING}

This work was supported by the National Institutes of Health Grant SC1DC015907 to AR-C. MD received the Company of Biologists traveling fellowship DEVTF-160703 and a "Bec.Ar" fellowship for a short stay supported by the Argentinian Government.

\section{ACKNOWLEDGMENTS}

We would like to acknowledge the Science Division Core Imaging Facility and Dr. Jonathan Levitt for providing access to a microtome to conduct histology procedures.

functional connectivity during wake, sleep, and anesthesia. Neurophotonics 6:035002.

Cadenas, L. T., Fischl, M. J., and Weisz, C. J. C. (2020). Synaptic inhibition of medial olivocochlear efferent neurons by neurons of the medial nucleus of the trapezoid body. J. Neurosci. 40, 509-525. doi: 10.1523/JNEUROSCI.1288-19. 2019

Cid, E., and de la Prida, L. M. (2019). Methods for single-cell recording and labeling in vivo. J. Neurosci. Methods 325:108354. doi: 10.1016/j.jneumeth.2019.108354

Clause, A., Kim, G., Sonntag, M., Weisz, C. J. C., Vetter, D. E., Rubsamen, R., et al. (2014). The precise temporal pattern of prehearing spontaneous activity 
is necessary for tonotopic map refinement. Neuron $82,822-835$. doi: 10.1016/j. neuron.2014.04.001

Crins, T. T., Rusu, S. I., Rodriguez-Contreras, A., and Borst, J. G. (2011). Developmental changes in short-term plasticity at the rat calyx of Held synapse. J. Neurosci. 31, 11706-11717. doi: 10.1523/JNEUROSCI.1995-11.2011

Di Guilmi, M. N., Boero, L. E., Castagna, V. C., Rodríguez-Contreras, A., Wedemeyer, C., Gómez-Casati, M. E., et al. (2019). Strengthening of the Efferent Olivocochlear System Leads to Synaptic Dysfunction and Tonotopy Disruption of a Central Auditory Nucleus. J. Neurosci. 39, 7037-7048. doi: 10.1523/JNEUROSCI.2536-18.2019

Elgoyhen, A. B., Johnson, D. S., Boulter, J., Vetter, D. E., and Heinemann, S. (1994). Alpha 9: an acetylcholine receptor with novel pharmacological properties expressed in rat cochlear hair cells. Cell 79, 705-715. doi: 10.1016/00928674(94)90555- $\mathrm{x}$

Elgoyhen, A. B., Vetter, D. E., Katz, E., Rothlin, C. V., Heinemann, S. F., and Boulter, J. (2001). Alpha10: a determinant of nicotinic cholinergic receptor function in mammalian vestibular and cochlear mechanosensory hair cells. Proc. Natl. Acad. Sci. U. S. A. 98, 3501-3506. doi: 10.1073/pnas.051622798

Glowatzki, E., and Fuchs, P. A. (2000). Cholinergic synaptic inhibition of inner hair cells in the neonatal mammalian cochlea. Science 288, 2366-2368. doi: $10.1126 /$ science. 288.5475 .2366

Golshani, P., Goncalves, J. T., Khoshkhoo, S., Mostany, R., Smirnakis, S., and Portera-Cailliau, C. (2009). Internally mediated developmental desynchronization of neocortical network activity. J. Neurosci. 29, 1089010899. doi: 10.1523/jneurosci.2012-09.2009

Gómez-Casati, M. E., Fuchs, P. A., Elgoyhen, A. B., and Katz, E. (2005). Biophysical and pharmacological characterization of nicotinic cholinergic receptors in rat cochlear inner hair cells. J. Physiol. 566, 103-118. doi: 10.1113/jphysiol.2005. 087155

Goodman, C. S., and Shatz, C. J. (1993). Developmental mechanisms that generate precise patterns of neuronal connectivity. Cell 72, 77-98. doi: 10.1016/s00928674(05)80030-3

Gribizis, A., Ge, X., Daigle, T. L., Ackman, J. B., Zeng, H., Lee, D., et al. (2019). Visual Cortex Gains Independence from Peripheral Drive before Eye Opening. Neuron 104, 711-723. doi: 10.1016/j.neuron.2019.08.015

Guinan, J. J., Warr, W. B., and Norris, B. E. (1983). Differential olivocochlear projections from lateral versus medial zones of the superior olivary complex. J. Comp. Neurol. 221, 358-370. doi: 10.1002/cne.902210310

Hanson, M. G., and Landmesser, L. T. (2004). Normal patterns of spontaneous activity are required for correct motor axon guidance and the expression of specific guidance molecules. Neuron 43, 687-701. doi: 10.1016/j.neuron.2004. 08.018

Hemmings, H. C., Akabas, M. H., Goldstein, P. A., Trudell, J. R., Orser, B. A., and Harrison, N. L. (2005). Emerging molecular mechanisms of general anesthetic action. Trends Pharmacol. Sci. 26, 503-510. doi: 10.1016/j.tips.2005.08.006

Johnson, S. L., Eckrich, T., Kuhn, S., Zampini, V., Franz, C., Ranatunga, K. M., et al. (2011). Position-dependent patterning of spontaneous action potentials in immature cochlear inner hair cells. Nat. Neurosci. 14, 711-717. doi: 10.1038/nn. 2803

Jones, T. A., Leake, P. A., Snyder, R. L., Stakhovskaya, O., and Bonham, B. (2007). Spontaneous discharge patterns in cochlear spiral ganglion cells before the onset of hearing in cats. J. Neurophysiol. 98, 1898-1908. doi: 10.1152/jn.00472.2007

Joshi, I., and Wang, L.-Y. (2002). Developmental profiles of glutamate receptors and synaptic transmission at a single synapse in the mouse auditory brainstem. J. Physiol. 540, 861-873. doi: 10.1113/jphysiol.2001.013506

Katz, E., Elgoyhen, A. B., Gomez-Casati, M. E., Knipper, M., Vetter, D. E., Fuchs, P. A., et al. (2004). Developmental regulation of nicotinic synapses on cochlear inner hair cells. J. Neurosci. 24, 7814-7820. doi: 10.1523/jneurosci.2102-04.2004

Kirkby, L. A., Sack, G. S., Firl, A., and Feller, M. B. (2013). A role for correlated spontaneous activity in the assembly of neural circuits. Neuron 80, 1129-1144. doi: 10.1016/j.neuron.2013.10.030

Kotak, V. C., and Sanes, D. H. (1995). Synaptically evoked prolonged depolarizations in the developing auditory system. J. Neurophysiol. 74, 16111620. doi: 10.1152/jn.1995.74.4.1611

Kros, C. J., Ruppersberg, J. P., and Rusch, A. (1998). Expression of a potassium current in inner hair cells during development of hearing in mice. Nature 394, 281-284. doi: $10.1038 / 28401$
Lipovsek, M., Im, G. J., Franchini, L. F., Pisciottano, F., Katz, E., Fuchs, P. A., et al. (2012). Phylogenetic differences in calcium permeability of the auditory hair cell cholinergic nicotinic receptor. Proc. Natl. Acad. Sci. U. S. A. 109, 4308-4313. doi: $10.1073 /$ pnas. 1115488109

Lippe, W. R. (1995). Relationship between frequency of spontaneous bursting and tonotopic position in the developing avian auditory system. Brain Res. 703, 205-213. doi: 10.1016/0006-8993(95)01096-3

Lorteije, J. A. M., Rusu, S. I., Kushmerick, C., and Borst, J. G. G. (2009). Reliability and precision of the mouse calyx of Held synapse. J. Neurosci. 29, 13770-13784. doi: 10.1523/jneurosci.3285-09.2009

MacDonald, J. F., Miljkovic, Z., and Pennefather, P. (1987). Use-dependent block of excitatory amino acid currents in cultured neurons by ketamine. J. Neurophysiol. 58, 251-266. doi: 10.1152/jn.1987.58.2.251

Maffei, L., and Galli-Resta, L. (1990). Correlation in the discharges of neighboring rat retinal ganglion cells during prenatal life. Proc. Natl. Acad. Sci. U. S. A. 87, 2861-2864. doi: 10.1073/pnas.87.7.2861

Marcotti, W., Johnson, S. L., Rusch, A., and Kros, C. J. (2003). Sodium and calcium currents shape action potentials in immature mouse inner hair cells. J. Physiol. 552, 743-761. doi: 10.1113/jphysiol.2003.043612

Mestre, H., Mori, Y., and Nedergaard, M. (2020). The Brain's Glymphatic System: current Controversies. Trends Neurosci. 43, 458-466. doi: 10.1016/j.tins.2020. 04.003

Moglie, M. J., Fuchs, P. A., Elgoyhen, A. B., and Goutman, J. D. (2018). Compartmentalization of antagonistic $\mathrm{Ca}(2+)$ signals in developing cochlear hair cells. Proc. Natl. Acad. Sci. U. S. A. 115, E2095-E2104.

Nicholson, C., Chen, K. C., Hrabětová, S., and Tao, L. (2000). Diffusion of molecules in brain extracellular space: theory and experiment. Prog. Brain Resin. 125, 129-154. doi: 10.1016/S0079-6123(00)25007-3

Petersen, C. C. H., Hahn, T. T. G., Mehta, M., Grinvald, A., and Sakmann, B. (2003). Interaction of sensory responses with spontaneous depolarization in layer 2/3 barrel cortex. Proc. Natl. Acad. Sci. U. S. A. 100, 13638-13643. doi: 10.1073/pnas.2235811100

Pinault, D. (1996). A novel single-cell staining procedure performed in vivo under electrophysiological control: morpho-functional features of juxtacellularly labeled thalamic cells and other central neurons with biocytin or Neurobiotin. J. Neurosci. Methods 65, 113-136. doi: 10.1016/0165-0270(95) 00144-1

Qiu, J., Singh, P., Pan, G., de Paolis, A., Champagne, F. A., Liu, J., et al. (2020). Defining the relationship between maternal care behavior and sensory development in Wistar rats: auditory periphery development, eye opening and brain gene expression. PLoS One 15:e237933. doi: 10.1371/journal.pone. 0237933

Rodríguez-Contreras, A., Shi, L., and Fu, B. M. (2014). A Method to Make a Craniotomy on the Ventral Skull of Neonate Rodents. J. Vis. Exp. 87, 51350. doi: $10.3791 / 51350$

Rodríguez-Contreras, A., van Hoeve, J. S. S., Habets, R. L. P., Locher, H., and Borst, J. G. G. (2008). Dynamic development of the calyx of Held synapse. Proc. Natl. Acad. Sci. U. S. A. 105, 5603-5608. doi: 10.1073/pnas.0801395105

Rothman, J. S., and Silver, R. A. (2018). NeuroMatic: an Integrated OpenSource Software Toolkit for Acquisition, Analysis and Simulation of Electrophysiological Data. Front. Neuroinform. 12:14. doi: 10.3389/fninf.2018. 00014

Rusu, S. I., and Borst, J. G. (2010). Developmental changes in intrinsic excitability of principal neurons in the rat medial nucleus of the trapezoid body. Dev. Neurobiol. 71, 284-295. doi: 10.1002/dneu.20856

Seabrook, T. A., Burbridge, T. J., Crair, M. C., and Huberman, A. D. (2017). Architecture, Function, and Assembly of the Mouse Visual System. Annu. Rev. Neurosci. 40, 499-538. doi: 10.1146/annurev-neuro-071714-033842

Sendin, G., Bourien, J., Rassendren, F., Puel, J. L., and Nouvian, R. (2014). Spatiotemporal pattern of action potential firing in developing inner hair cells of the mouse cochlea. Proc. Natl. Acad. Sci. U. S. A. 111, 1999-2004. doi: 10.1073/pnas.1319615111

Shi, L., and Rodríguez-Contreras, A. (2016)."In vivo two-photon imaging measuring the blood-brain barrier permeability during early postnatal brain development in rodent," in Proc. SPIE 9712, Multiphoton Microscopy in the Biomedical Sciences XVI, eds A. Periasamy, P. T. C. So, and K. König (Washington: SPIE), doi: 10.1117/12.2219571 
Sierksma, M. C., and Borst, J. G. G. (2017). Resistance to action potential depression of a rat axon terminal in vivo. Proc. Natl. Acad. Sci. U. S. A. 114, 4249-4254. doi: $10.1073 /$ pnas.1619433114

Simmons, D. D., Mansdorf, N. B., and Kim, J. H. (1996). Olivocochlear innervation of inner and outer hair cells during postnatal maturation: evidence for a waiting period. J. Comp. Neurol. 370, 551-562. doi: 10.1002/(sici)1096-9861(19960708) 370:4<551::aid-cne10>3.0.co;2-m

Sonntag, M., Englitz, B., Kopp-Scheinpflug, C., and Rubsamen, R. (2009). Early postnatal development of spontaneous and acoustically evoked discharge activity of principal cells of the medial nucleus of the trapezoid body: an in vivo study in mice. J. Neurosci. 29, 9510-9520. doi: 10.1523/jneurosci.1377-09.2009

Taschenberger, H., and von Gersdorff, H. (2000). Fine-tuning an auditory synapse for speed and fidelity: developmental changes in presynaptic waveform, EPSC kinetics, and synaptic plasticity. J. Neurosci. 20, 9162-9173. doi: 10.1523/ JNEUROSCI.20-24-09162.2000

Tritsch, N. X., Rodríguez-contreras, A., Crins, T. T. H., Wang, H. C., Borst, J. G., Bergles, D. E., et al. (2010). Calcium action potentials in hair cells pattern auditory neuron activity before hearing onset. Nat. Neurosci. 13, 1050-1052. doi: $10.1038 / \mathrm{nn} .2604$

Tritsch, N. X., Yi, E., Gale, J. E., Glowatzki, E., and Bergles, D. E. (2007). The origin of spontaneous activity in the developing auditory system. Nature 450, 50-55. doi: $10.1038 /$ nature 06233

van der Heijden, M., Lorteije, J. A. M., Plauska, A., Roberts, M. T., Golding, N. L., and Borst, J. G. G. (2013). Directional hearing by linear summation of binaural inputs at the medial superior olive. Neuron 78, 936-948. doi: 10.1016/j.neuron. 2013.04.028

Wang, H. C., Lin, C. C., Cheung, R., Zhang-Hooks, Y., Agarwal, A., Ellis-Davies, G., et al. (2015). Spontaneous Activity of Cochlear Hair Cells Triggered by Fluid Secretion Mechanism in Adjacent Support Cells. Cell 163, 1348-1359. doi: $10.1016 /$ j.cell.2015.10.070

Wang, Y., Sanghvi, M., Gribizis, A., Zhang, Y., Song, L., Morley, B., et al. (2020). Efferent feedback enforces bilateral coupling of spontaneous activity in the developing auditory system. bioRxiv [Preprint]. doi: 10.1101/2020.08.12. 248799

Wang, H.-Y., Eguchi, K., Yamashita, T., and Takahashi, T. (2020). FrequencyDependent Block of Excitatory Neurotransmission by Isoflurane via Dual Presynaptic Mechanisms. J. Neurosci. 40, 4103-4115. doi: 10.1523/ JNEUROSCI.2946-19.2020

Warr, W. B. (1975). Olivocochlear and vestibular efferent neurons of the feline brain stem: their location, morphology and number determined by retrograde axonal transport and acetylcholinesterase histochemistry. J. Comp. Neurol. 161, 159-181. doi: 10.1002/cne.901610203

Warr, W. B., and Guinan, J. J. (1979). Efferent innervation of the organ of corti: two separate systems. Brain Res. 173, 152-155. doi: 10.1016/0006-8993(79) 91104-1

Weisstaub, N., Vetter, D. E., Belén Elgoyhen, A., and Katz, E. (2002). The $\alpha 9 \alpha 10$ nicotinic acetylcholine receptor is permeable to and is modulated by divalent cations. Hear. Res. 167, 122-135. doi: 10.1016/S0378-5955(02) 00380-5

Wu, X. S., Sun, J. Y., Evers, A. S., Crowder, M., and Wu, L. G. (2004). Isoflurane inhibits transmitter release and the presynaptic action potential. Anesthesiology 100, 663-670. doi: 10.1097/00000542-20040300000029

Conflict of Interest: The authors declare that the research was conducted in the absence of any commercial or financial relationships that could be construed as a potential conflict of interest.

Copyright (c) 2021 Di Guilmi and Rodríguez-Contreras. This is an open-access article distributed under the terms of the Creative Commons Attribution License (CC BY). The use, distribution or reproduction in other forums is permitted, provided the original author(s) and the copyright owner(s) are credited and that the original publication in this journal is cited, in accordance with accepted academic practice. No use, distribution or reproduction is permitted which does not comply with these terms. 